\title{
RELIABLE MINIMUM ENERGY COST ROUTING ALGORITHM TO FIND THE ENERGY-EFFICIENT AND RELIABLE ROUTES CONSIDERING RESIDUAL ENERGY IN WIRELESS AD-HOC NETRWORKS
}

\author{
Mallashetty C Tavade ${ }^{1}$, Girish V.A ${ }^{2}$ \\ ${ }^{1}$ P.G.Scholar ECE Dept. Dayananda Sagar College of Engineering Bangaluru, India \\ ${ }^{2}$ Professor and Head ECE dept. Dayananda Sagar College of Engineering Bangaluru, India
}

\begin{abstract}
In this paper we proposed Reliable Minimum Energy Cost Routing (RMECR) for wireless Ad-hoc networks (WANET). RMECR will select the route which is consuming less energy, route which have high reliability, high quality and also important factor is RMECR considers the remaining battery energy of the nodes there by increases the lifetime of the network to the greater extent. Simulation studies shows that RMECR is able to find the reliable and energy efficient route like RMER[12] but major factor which RMECR considers is the remaining battery energy of the nodes thereby increasing the network lifetime.
\end{abstract}

Keywords: RMER, RMECR, WANET, Ad-Hoc-Network.

\section{INTRODUCTION}

Wireless Ad-Hoc networks (WANET) are infrastructure less networks that means they neither have base station for transmitting the data nor they have access points like routers in wireless sensor networks to route the data from one point to other point. In WANET nodes are assembled with transreceiver units, battery, sensors, processors so nodes in WANET perform the job of base station and access points as in the case of wireless sensor networks, so for performing these operations the node undergoes problems like battery power consumption, power consumed by transmitting unit for transmitting the packet and power consumed by receiver unit to receive the acknowledgement from destination node . In previous studies of ETX metric[1] was found the estimated transmission count for all the possible paths which connect form source to destination node and selects the path which has high ETX ratio, in this approach we are considering only the reliability of links, not considering the energy-efficiency and the residual battery power of nodes so this will lead to the decrease in lifetime of wireless Ad-Hoc Networks, so in our paper we are going to introduce two novel algorithms called Reliable Minimum Energy Routing (RMER)[12] and Reliable minimum energy cost routing (RMECR) [3,4]. RMER algorithm takes into account the minute factors such as transreceiver unit power, reliability of link to find out the weight of each link between source and destination node and selects the link which has minimum weight to transmit the data packet [5]. In RMER[12] we are finding the route which is energy efficient and reliable but if we go on using the same routes all the time the intermediate nodes which are responsible for transmitting the packet are being over used hence there is reduction in battery life of nodes and hence after some time they will become dead so this indirectly effects the lifetime of Ad-Hoc networks hence another algorithm RMECR is used which considers the remaining battery of nodes to transmit the packet along with energy-efficiency and reliability $[1,2,3,4,5,6]$. This motivated us to study RMECR algorithm with different parameters from the traditional existing work.

\section{PRILIMINARIES}

\subsection{Network Model}

We represent the topology of wireless Ad-Hoc network as $\mathrm{G}(\mathrm{v}, \mathrm{e})$ where $\mathrm{v}$ and $\mathrm{e}$ represents the set of nodes and links(edges), respectively. Each node in the WANET are represented by a unique identifier between 1to N, Nodes are assumed to be battery powered the remaining battery power is represented by $C_{u}$. The link in the network is represented by $(\mathrm{u}, \mathrm{v})$ where $\mathrm{u}$ and $\mathrm{v}$ represent the transmitting node and receiving node respectively, we denoting the error free packet delivery ratio $\mathrm{x}$ bits from node $\mathrm{u}$ to node $\mathrm{v}$ by $\mathrm{P}_{\mathrm{u}, \mathrm{v}}(\mathrm{x})$. while transmitting the data packet from node $\mathrm{u}$ to $\mathrm{v}$ we are considering minimum power from a set of power to achieve energy efficiency. There will be one hop between two nodes so mathematically we can show there are totally $\left(\mathrm{n}_{1+} \mathrm{n}_{2 \ldots} \ldots \mathrm{n}_{\mathrm{h} . .} \mathrm{n}_{\mathrm{h}+1)}\right.$ nodes present . where $\mathrm{n}_{1}$ is the source node and $n_{h+1}$ is the destination node and rest of the nodes are intermediate nodes which are useful for transmitting the packet hop by hop.

\subsection{Art of Nodes in Wireless Ad-Hoc Network}

Wireless ad-hoc networks typically refers to any set of networks where all devices within network have equal status 
and can be connected to other network devices which are within link range or coverage area. The nodes in the wireless ad-hoc networks are rapidly and randomly deployed each node in WANET(wireless ad-hoc networks) consist of its own power source, battery, processing unit and RF transreceiver for transmitting and receiving the data packets. As it is a autonomous network no base station no routers are required, nodes only have to find their neighbors who are within the range and they only have to transmit the data packet on their own. All nodes in WANET rely on batteries or any other exhaustible means for their energy so, to increase the network lifetime the efficient system or network design criteria must first optimize the energy conservation.

\subsection{Energy Consumption for Packet Transmission in WANET}

Consider that we are transmitting the $\mathrm{x}$ bits of data packet from transmitting node $\mathrm{u}$ to receiving node $\mathrm{v}$ through link $(\mathrm{u}, \mathrm{v})$, to transmit the $\mathrm{x}$ bits of data packet from node $\mathrm{u}$ definitely the transmitting unit of node $u$ is consuming some power $\mathrm{p}(\mathrm{u}, \mathrm{v})$ and also processing unit consumes some power lets denote it is $A_{u}$ similarly the receiver unit of node $v$ which is receiving the data packet also consumes some power $\mathrm{B}_{\mathrm{v}}, 0<\mathrm{k}<1$ is the power efficiency of the power amplifier of node $\mathrm{u}, \mathrm{r}$ bits /sec is the data rate of the link from node $\mathrm{u}$ to node $\mathrm{v}$. this $\mathrm{r}$ bits/sec value we are finding using previously developed ETX metric approach. So using all these parameters we can find the energy consumed $e(x)$ by transmitting node $u$ as

$$
e(x)=\left(A_{u}+\frac{P_{u, v}}{K}\right) \frac{x}{r} \forall x \geq 0
$$

An energy consumed by receiver unit is given by

$$
\mathrm{r}_{\mathrm{u}, \mathrm{v}}(\mathrm{x})=\left(\frac{B_{v}}{r}\right) x \forall x \geq 0
$$

\subsection{Consideration of Energy for Reliable Routing}

Our main objective is to find the reliable route which minimizes the energy consumption of a path, since energy consumes and reliability are related to one another, if the path is reliable then there is no necessity of retransmission of data packet again and again so energy consumed for transmitting the data packet will be less, and in the case of unreliable routes the loss of packet is more so retransmission of packet increases which leads to more energy consumption - so in our paper we are introducing two energy aware reliable routing algorithms for $\mathrm{HBH}$ and E2E systems, they are called Reliable Minimum Energy Cost routing (RMER) and Reliable Minimum Energy Cost Routing (RMECR). RMER finds the energy consumed by nodes for transmitting the packet to the destination and RMECR finds the expected battery cost of nodes to transmit the packet from source to destination.

\section{PROPOSED APPROACH}

In the proposed approach RMECR algorithm is developed, the RMECR addresses three important requirements of ad hoc networks i.e. energy-efficiency, reliability, and prolonging network lifetime. It considers the energy consumption and the remaining battery energy of nodes as well as quality of links to find energy-efficient and reliable routes that increase the operational lifetime of the network. RMER, on the other hand, is an energy-efficient routing algorithm which finds routes minimizing the total energy required for end-to-end packet traversal. RMECR are proposed for networks in which either hop-by-hop or endto-end retransmissions ensure reliability.

\subsection{Reliable Minimum Energy Cost Routing (RMECR)}

RMER considers both reliability and energy efficiency of the link between two nodes before transmitting the data packet but it will not consider the remaining battery life of the nodes hence indirectly it leads to the degradation in lifetime of wireless Ad-Hoc network. This drawback is overcome in next algorithm called RMECR. RMECR algorithm is build on top of RMER algorithm. It considers even cost of the network, reliability as well as the residual battery power of the nodes in the network. The link weight of the RMECR algorithm can be described as follows [8]

$$
\begin{aligned}
& W_{R M E C R}(u, v)=\frac{L_{d}}{r}\left(\frac{A_{u}+\frac{P_{u, v}}{k_{u}}}{C_{u}}+\frac{B_{v}}{C_{v}}\right)_{(2)} \\
& +\frac{L_{e}}{r}\left(\frac{A_{v}+\frac{P_{v, u}}{k_{v}}}{C_{v}}+\frac{B_{u}}{C_{u}}\right)
\end{aligned}
$$

Where, $\mathrm{Cu}$ and $\mathrm{Cv}$ energy cost of the path from Node ' $\mathrm{u}$ ' to Node ' $v$ ' and energy cost of the path from Node ' $v$ ' to ' $u$ ' respectively. The energy cost of the path from Node $u$ to Node $\mathrm{v}$ and back is given by [9]

$$
\begin{aligned}
& C(P(u, v))=N_{p}\left(L_{d}\right) * R_{u}\left(L_{d}\right) * e_{u, v}\left(L_{d}\right)+M_{p}\left(L_{e}\right) \\
& * R_{v}\left(L_{e}\right) * e_{v, u}\left(L_{e}\right)
\end{aligned}
$$

Where, $\mathrm{L}_{\mathrm{d}}=$ Packet length send from Node ' $\mathrm{u}$ ' to Node ' $v$ ', $\mathrm{r}=$ data rate of the link, $\mathrm{A}_{\mathrm{u}}=$ power required by the transmitting unit at Node ' $\mathrm{u}$ ', $\mathrm{B}_{\mathrm{v}}=$ power required by the transmitting unit at Node ' $\mathrm{V}$ ', $\mathrm{P}_{\mathrm{u}, \mathrm{v}}=$ power required for transmission from ' $u$ ' to ' $v$ ', $K_{u}=$ propagation efficiency of Node $\mathrm{u} \leq \mathrm{K}_{\mathrm{u}} \leq 1, \mathrm{~L}_{\mathrm{e}}=$ packet length (ACK) send from Node ' $\mathrm{v}$ ' to Node ' $\mathrm{u}$ ', $\mathrm{A}_{\mathrm{v}}=$ power required by the transmitting unit at Node ' $v$ ', $B_{u}=$ power required by the transmitting unit at Node ' $\mathrm{u}$ ', $\mathrm{P}_{\mathrm{v}, \mathrm{u}}=$ power required for transmission from ' $\mathrm{v}$ ' to ' $\mathrm{u}$ ', $\mathrm{K}_{\mathrm{v}}=$ propagation efficiency of Node $\mathrm{v} \leq \mathrm{K}_{\mathrm{v}} \leq 1$. 
$\mathrm{N}_{\mathrm{p}}\left(\mathrm{L}_{\mathrm{d}}\right)=$ Expected transmission count packet send from Node ' $u$ ' to Node ' $v$ ', $\mathrm{R}_{\mathrm{u}}\left(\mathrm{L}_{\mathrm{d}}\right)=$ Reliability of the link from Node ' $u$ ' to Node ' $v$ ', $e_{u, v}\left(L_{d}\right)=$ Energy require for transmitting packet of length Ld form Node ' $u$ ' to Node ' $v$ ', $\mathrm{Mp}(\mathrm{Ls})=$ Expected transmission count ACK packet send from Node ' $v$ ' to Node ' $u$ ', $\mathrm{R}_{\mathrm{v}}\left(\mathrm{L}_{\mathrm{e}}\right)=$ Reliability of the link from Node ' $v$ ' to Node ' $u$ ', $e_{v, u}\left(L_{e}\right)=$ Energy require for transmitting packet of length Le form Node ' $v$ ' to Node ' $u$ '.

The Reliability of the Forward Link is given by the following equation [10].

$$
\begin{aligned}
& R_{u, v}\left(L_{d}\right)=1-\operatorname{Pr}\left\{\text { packet lost after } Q_{u} \text { transmissions }\right\} \\
& =1-\left[1-p_{u, v}\left(L_{d}\right)\right]^{Q_{u}}
\end{aligned}
$$

The Reliability of the Backward Link is given by the following equation [11],

\section{$R_{v, u}\left(L_{d}\right)=1-\operatorname{Pr}\left\{\right.$ packet lost after $Q_{u}$ transmissions $\}$}

$=1-\left[1-p_{v, u}\left(L_{d}\right)\right]^{Q_{u}}$

The energy consumed by the transmitting node $u$ is given by

$$
e_{u, v}=E\left[n_{u, v}\left(L_{d}\right)\right] \xi_{u, v}\left(L_{d}\right)
$$$$
+E\left[m_{v, u}\left(L_{d}\right)\right] \operatorname{erx} x_{v, u}\left(L_{h}\right)
$$

\section{RESULTS AND DISCUSSION}

The results for RMECR as shown below, Here as we can see in the fig 1 nodes are deployed randomly to form a wireless Ad-Hoc network, for the sake of understanding at the time of simulation we have selected the source node as 74 and destination node as 75. As we have mentioned earlier RMECR finds link reliability of all nodes in the WANET then it finds the neighbor nodes of the source node i.e in our case source node is 74 ,from fig 3 it is clear that among neighbor nodes of 74 , node having id 27 is having minimum weight and also high reliability as shown in fig 2 so node 74 selects node 27 as next neighbor ,form the RMECR weight equation we can make out that weight of link is inversely proportional to the remaining battery power of node $\mathrm{C}_{\mathrm{u}}$ or $\mathrm{C}_{\mathrm{v}}$ this procedure continues till the destination node is reached. We are also calculating the number of hops required for transmitting the data from source node $t$ destination node as shown in fig 6, we are calculating route discovery time and energy consumed for finding the route between source node and destination node as shown in fig 7 and fig 8 respectively.

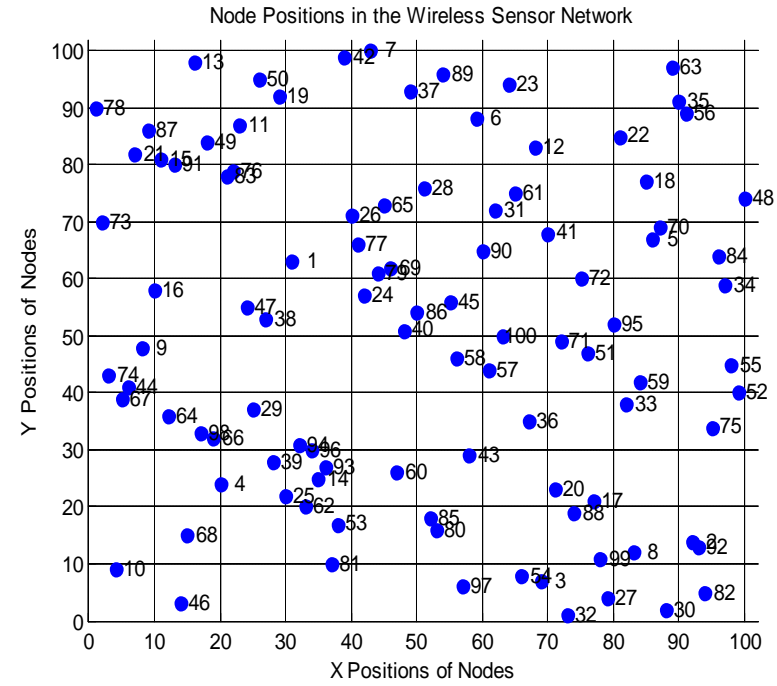

Fig: 1

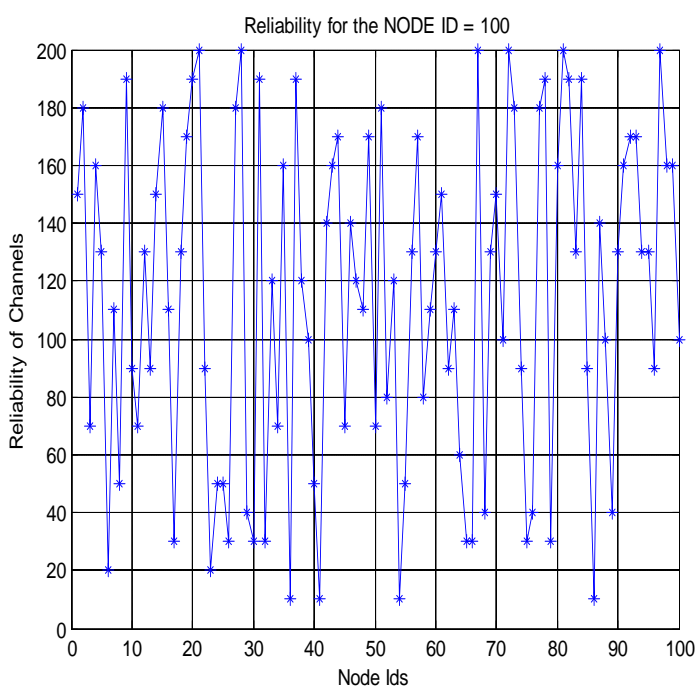

Fig: 2

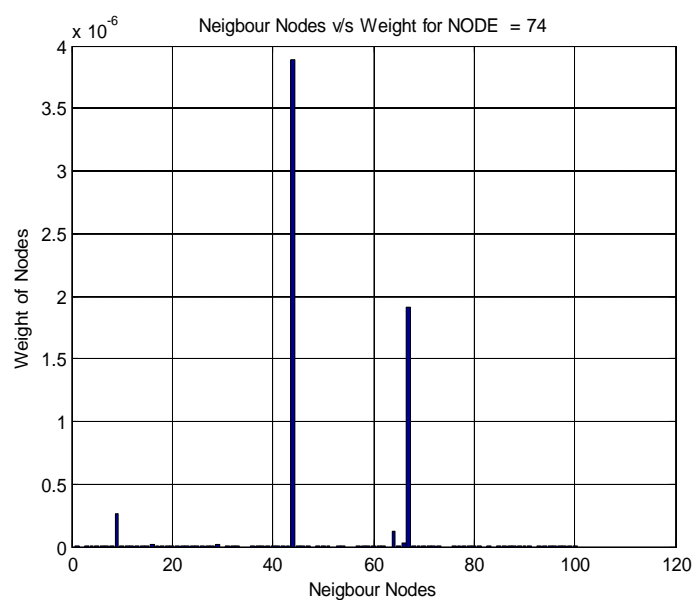

Fig: 3 


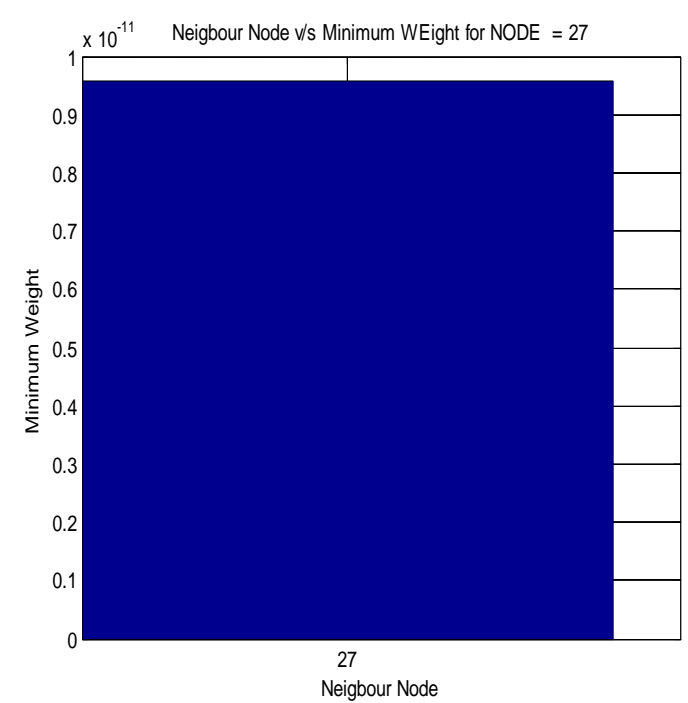

Fig: 4

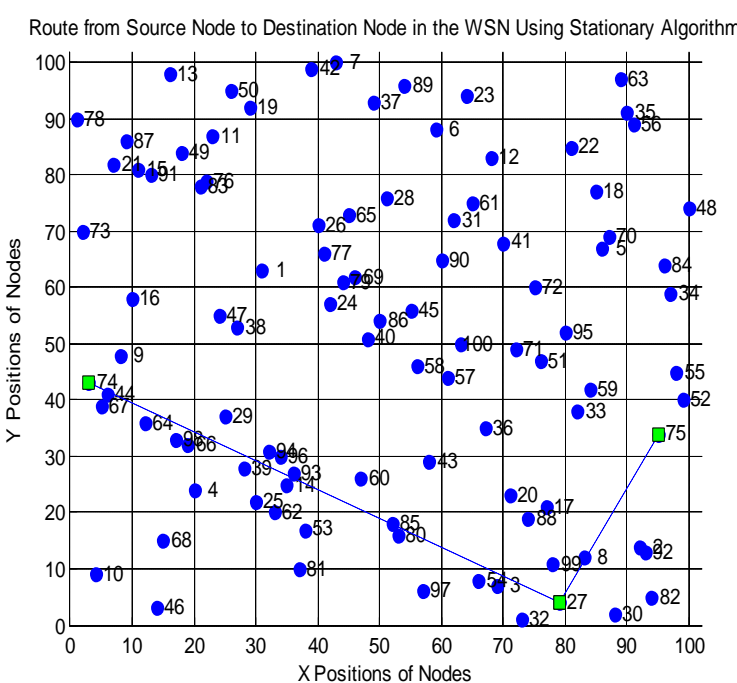

Fig: 5

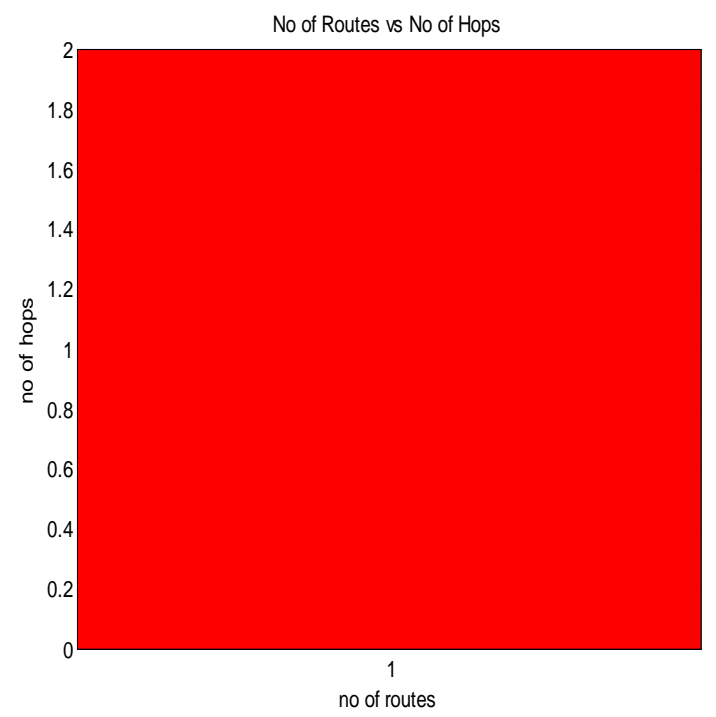

Fig: 6

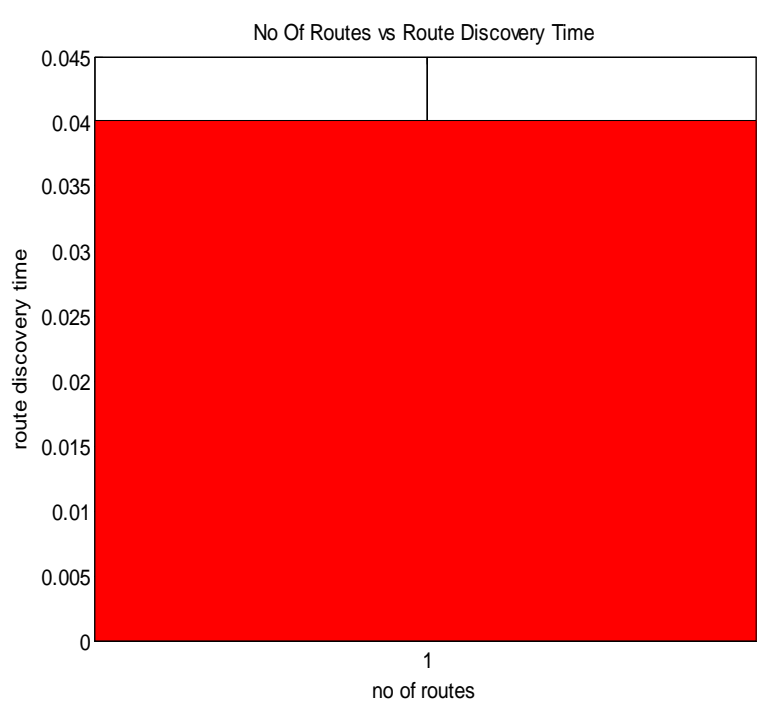

Fig: 7

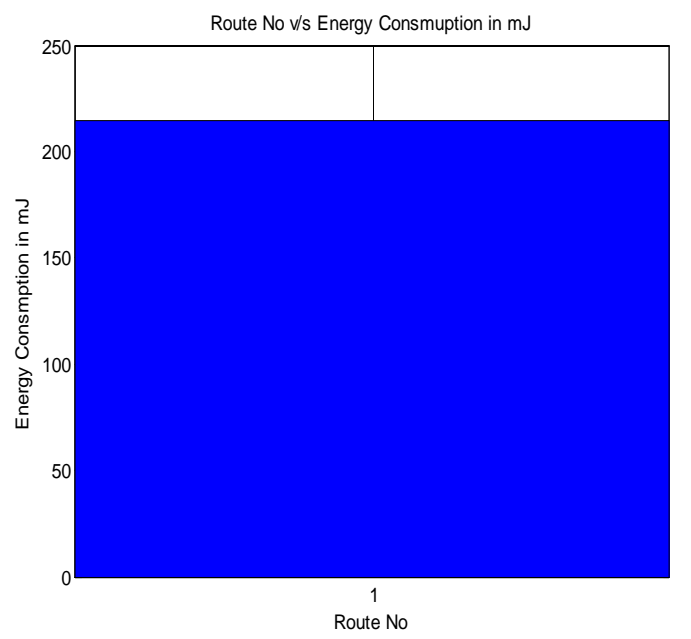

Fig: 8

\section{CONCLUSION}

We have successfully simulated Reliable Minimum Energy Cost Routing (RMECR) algorithm for wireless Ad-hoc networks (WANET). RMECR will select the route which is consuming less energy, route which have high reliability, high quality and also important factor is RMECR considers the remaining battery energy of the nodes there by increases the lifetime of the network to the greater extent. Simulation shows that RMECR is able to find the reliable and energy efficient route like RMER[12] but major factor which RMECR considers is the remaining battery energy of the nodes, since from weight equation of RMECR we can see weight is inversely proportional to the residual battery energy of the node .thereby increasing the network lifetime.

\section{REFERENCES}

[1]. D.S.J. De Couto, D. Aguayo, J. Bicket, and R.Morris, "A High-Throughput Path Metric for Multi-Hop Wireless Routing," Proc. ACM MobiCom, pp. 134- 146, 2003. 
[2]. S. Singh and C. Raghavendra, "Power Aware MultiAccess Protocol with Signaling for Ad Hoc Networks,"ACM Computer Comm. Rev., vol. 28, pp. 5-26, 1999.

[3]. J. Gomez, A.T. Campbell, M. Naghshineh, and C.Bisdikian, "PARO: Supporting Dynamic Power Controlled Routing in Wireless Ad Hoc Networks," Wireless Networks, vol. 9, no. 5, pp. 443-460, 2003.

[4]. S. Banerjee and A. Misra, "Minimum Energy Paths for Reliable Communication in Multi-HopWireless Networks," Proc. ACM MobiHoc, pp. 146-156, June 2002.

[5]. Q. Dong, S. Banerjee, M. Adler, and A. Misra, "Minimum Energy Reliable Paths Using Unreliable Wireless Links," Proc. ACM MobiHoc, pp. 449-459, May 2005.

[6]. X.-Y. Li, Y. Wang, H. Chen, X. Chu, Y. Wu, and Y. Qi, "Reliable and Energy-Efficient Routing for Static Wireless Ad Hoc Networks with Unreliable Links," IEEE Trans. Parallel and Distributed Systems, vol. 20, no. 10, pp. 14081421, Oct. 2009.

[7]. X. Li, H. Chen, Y. Shu, X. Chu, and Y.-W. Wu, "Energy Efficient Routing with Unreliable Links in Wireless Networks," Proc. IEEE Int'l Conf. Mobile Adhoc and Sensor Systems (MASS '06), pp. 160-169, 2006

[8]. S. Singh, M. Woo, and C.S. Raghavendra, "PowerAware Routing in Mobile Ad Hoc Networks," Proc. ACM MobiCom, Oct. 1998.

[9]. C. Toh, "Maximum Battery Life Routing to Support Ubiquitous Mobile Computing in Wireless Ad Hoc Networks," IEEE Comm. Magazine, vol. 39, no. 6, pp. 138147, June 2001.

[10]. D. Kim, J.J.G. Luna Aceves, K. Obraczka, J. Carlos Cano, and P. Manzoni, "Routing Mechanisms for Mobile Ad Hoc Networks Based on the Energy Drain Rate," IEEE Trans. Mobile Computing, vol. 2, no. 2, pp. 161-173, Apr.June 2003.

[11]. J.-H. Chang and L. Tassiulas, "Maximum Lifetime Routing in Wireless Sensor Networks," IEEE/ACM Trans. Networking, vol. 12, no. 4, pp. 609-619, Aug. 2004

[12]. J. Vazifehdan, R. Prasad, and I. Niemegeers, "Minimum Battery Cost Reliable Routing in Ad Hoc Wireless Networks," Proc. Eighth IEEE Consumer Comm. and Networking Conf., Jan. 2011. 